\title{
Study on Dynamical Behavior in Complex Network with Community
}

\section{Structure}

\author{
Xuefeng Liang \\ School of Mathematic and statistics, Tianshui Normal University, Tianshui, 741001 \\ langxf @163.com
}

\begin{abstract}
The impact of community structure on synchronization behavior of oscillatory complex network is investigated, focusing on the community number. Numerical simulations show that the bigger community number, the stronger synchronizability of power network will be. That is, the synchronizability of the network is significantly influenced by community numbers.
\end{abstract}

Keywords: Complex network, Community structure, Synchronizability

\section{Introduction}

The synchronization process of large populations of weakly coupled oscillators often appears in various systems, and it has been extensively studied in different scientific communities such as physics, biology, sociology, etc. [1]. Power network is generally formed by a large number of oscillators with the purpose is to deliver power from generators to final consumers [2-4]. A node in power network is defined to be a point at which power is injected by a generator or extracted by consumers, or to other points. A link is then defined as a connection between any a pair of such nodes. It has been demonstrated that many real world networks show community structure, such as power network [5], communication networks [6], and so on. To the best of our knowledge, synchronization of power network with community structure has not been previously investigated. So it is interesting to study the synchronization of power network with community structure.

\section{Complex network model}

Each node is characterized by the same equation of motion with the electric power $P_{j}$, where it generates power (when $P_{j}>0$ ) or consumes power (when $P_{j}<0$ ). The phase of each element is then described as

$$
\theta_{j}(t)=\Omega t+\phi_{j}(t)
$$

The equation of motion for the phase deviation $\phi_{j}(t)$ is obtained:

$$
P_{\text {source }, j}=P_{\text {diss }, j}+P_{\text {acc }, j}+P_{\text {trans }, j} .
$$

Because of the rotation of the mechanical rotors, energy is dissipated

$$
P_{\text {diss }, j}=k\left(\theta_{j}\right)^{2},
$$


The kinetic energy accumulated is described as following:

$$
P_{a c c, j}=\frac{1}{2} I \frac{d}{d t}\left(\theta_{j}\right)^{2},
$$

And the power transmitted from element $i$ to $j$ is written as following:

$$
P_{\text {trans }, i j}=P_{\mathrm{max}, i j} \sin \left(\theta_{i}-\theta_{j}\right) .
$$

Inserting equations (3-5) into equation (2) one gets:

$$
P_{\text {source }, j} \cong I \phi_{j} \phi_{j}+k\left(\phi_{j}\right)^{2}-\sum_{i=1}^{N} P_{\max , i j} \sin \left(\phi_{i}-\phi_{j}\right)
$$

One finds the equation of motion

$$
P_{\text {source }, j} \cong I \Omega \phi_{j}+k \Omega^{2}+\left[I \phi_{j}+2 k \Omega\right] \phi_{j}-\sum_{i=1}^{N} P_{\text {max }, i j} \sin \left(\phi_{i}-\phi_{j}\right) .
$$

We will neglect the term proportional to the acceleration;

$$
\phi_{j}=\frac{2 k \Omega}{I}
$$

Under this hypothesis equation (7) becomes:

$$
I \Omega \phi_{j} \cong P_{\text {source }, j}-k \Omega^{2}-2 k \Omega \phi_{j}+\sum_{i=1}^{N} P_{\max , i j} \sin \left(\phi_{i}-\phi_{j}\right) .
$$

Such equation can be described as:

$$
\phi_{j}=P_{j}-\alpha \phi_{j}+K \sum_{i=1}^{N} a_{i j} \sin \left(\phi_{i}-\phi_{j}\right)
$$

\section{Synchronization analysis of power network}

A skecth is shown in Fig.1, where the big circle is the community of power network, the small diagrams in circle means the nodes of power network.

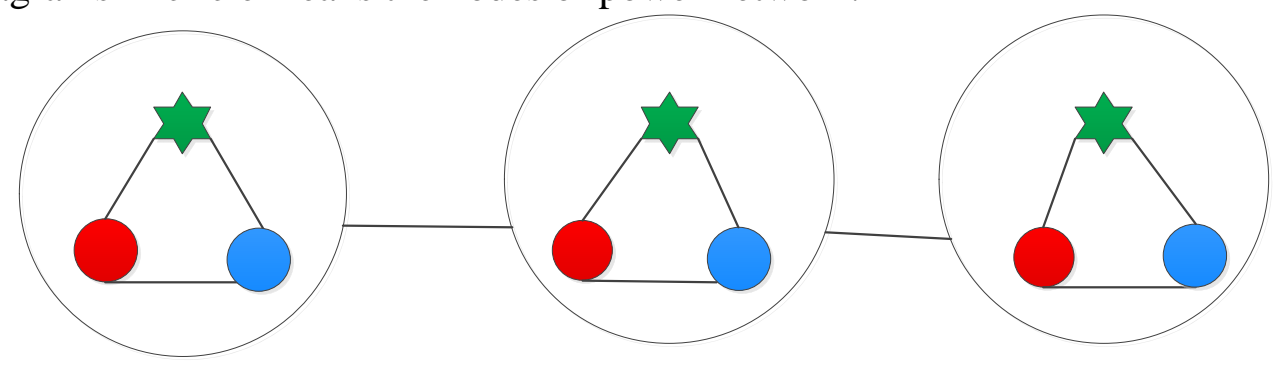

Fig.1 The topology of chain community network

The community numbers vary from less to more. A comparsion study between different numbers of communities is shown in Fig.2. 


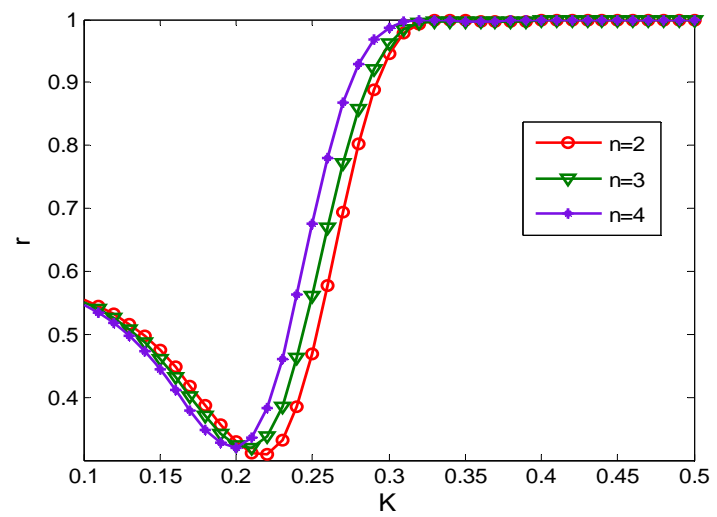

Fig.2 Order parameter $r$ vs coupling strength $K$ for different community number

Figure 2 depicts how the community number impact the synchronizability of power network with fixed community strength. From Fig.2, it can be seen that the order parameter $r$ with $n=2$ is smaller than $r$ with $n=3$ and $n=4$. Namely, the bigger community number, the stronger synchronizability of power network will be.

\section{The total node numbers are unchanged}

In the following, adjust the numbers of community to study the performance of order parameter.

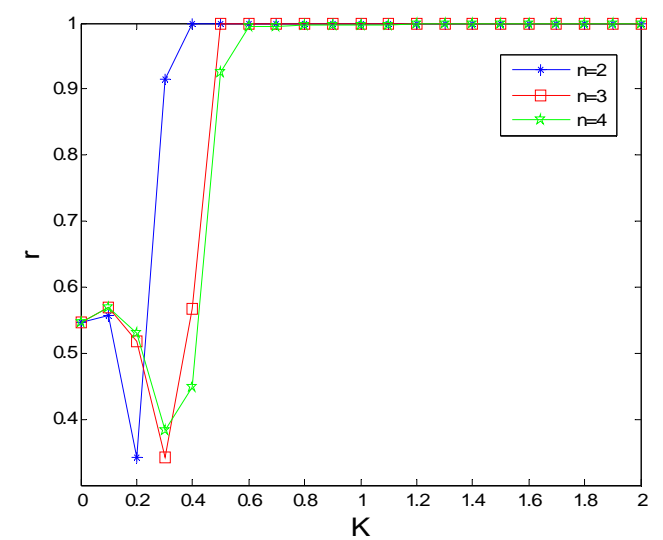

Fig.3 Order parameter $r$ vs coupling strength $K$ for different community number $n$.

One can see that order parameter is larger with the decreasing of community numbers. So the community number plays a positive role on the synchronization of complex network.

\section{Conclusion}

This paper investigates the influence of community structure on synchronization for a power network on coarse scales. Focusing on the community number, we analyze the effects of community structure on the synchronizability of power network. As for power network with fixed community strength, it is revealed the bigger community number, the stronger synchronizability of power network will be. 


\section{References}

[1] L.M. Pecora, T.L. Carrol: Synchronization in chaotic systems. Phys. Rev. Lett. 64(1990) 821-826.

[2] G. Filatrella, A.H. Nielsen, N.F. Pedersen: Analysis of a power grid using a Kuramoto-like model. EPJB 61(2008)485-491

[3] P. Seliger, S.C.Young, L.S. Tsimring: Plasticity and learning in a network of coupled phase oscillators. Phys. Rev. E 65(2002)041906.

[4] R. Carareto, M. S. Baptista, C. Grebogi: Natural synchronization in power-grids with anti-correlated units 18 (2013) 1035-1046

[5] F. Dorfler and F. Bullo: Synchronization and transient stability in power networks and non-uniform Kuramoto oscillators. Proc. American Control Conf(2010) 930-937.

[6] P. Pourbeik, P.S. Kundur, C.W. Taylor: The anatomy of a power grid blackout-root causes and dynamics of recent major blackouts, Power and Energy Magazine, IEEE 4(2006)22-26. 